\title{
You are more than what you eat: potentially adaptive enrichment of microbiome functions across bat dietary niches
}

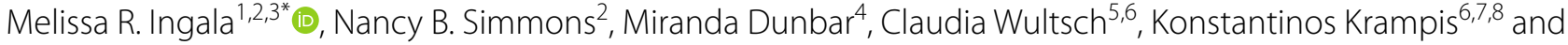 \\ Susan L. Perkins 3,5
}

\begin{abstract}
Background: Animals evolved in a microbial world, and their gut microbial symbionts have played a role in their ecological diversification. While many recent studies report patterns of phylosymbiosis between hosts and their gut bacteria, fewer studies examine the potentially adaptive functional contributions of these microbes to the dietary habits of their hosts. In this study, we examined predicted metabolic pathways in the gut bacteria of more than 500 individual bats belonging to 60 species and compare the enrichment of these functions across hosts with distinct dietary ecologies.
\end{abstract}

Results: We found that predicted microbiome functions were differentially enriched across hosts with different diets. Using a machine-learning approach, we also found that inferred microbiome functions could be used to predict specialized host diets with reasonable accuracy. We detected a relationship between both host phylogeny and diet with respect to microbiome functional repertoires. Because many predicted functions could potentially fill nutritional gaps for bats with specialized diets, we considered pathways discriminating dietary niches as traits of the host and fit them to comparative phylogenetic models of evolution. Our results suggest that some, but not all, predicted microbiome functions may evolve toward adaptive optima and thus be visible to the forces of natural selection operating on hosts over evolutionary time.

Conclusions: Our results suggest that bats with specialized diets may partially rely on their gut microbes to fulfill or augment critical nutritional pathways, including essential amino acid synthesis, fatty acid biosynthesis, and the generation of cofactors and vitamins essential for proper nutrition. Our work adds to a growing body of literature suggesting that animal microbiomes are structured by a combination of ecological and evolutionary processes and sets the stage for future metagenomic and metabolic characterization of the bat microbiome to explore links between bacterial metabolism and host nutrition.

Keywords: Bats, Microbiome, Functional prediction, Dietary ecology, Evolution, 165 rRNA gene

*Correspondence: ingala.melissar@gmail.com

${ }^{1}$ Department of Vertebrate Zoology, National Museum of Natural History, Washington, DC, USA

Full list of author information is available at the end of the article

\section{Background}

Host-microbe interactions have shaped the ecological and evolutionary history of life on Earth, and there is growing evidence that many animals have adapted to their diets through a combination of physiological adaptations and metabolic pathways encoded by the gut microbiome [1-3]. As a result, many vertebrate groups show gut microbiomes whose taxonomic compositions 
closely mirror host evolutionary history and dietary strategies [4-6]. Because host diet and evolutionary history are themselves often correlated (i.e., closely related species may share similar diets), it can be challenging to parse the relationship between host diet and evolutionary history in influencing microbiome composition, leaving little consensus on which force is the primary driver in patterning the gut microbiome and whether the strength of these forces varies among host clades [7, 8]. In addition, it can be difficult to extrapolate potentially adaptive functions of animal microbiomes by testing for phylosymbiosis, or the recapitulation of host phylogeny in bacterial community similarity, alone. The majority of studies testing for phylosymbiosis consider only bacterial taxonomy and do not explicitly test any functional hypotheses [but see 6-8]. Because bacterial communities are characterized by a high rate of functional redundancy, phylogenetically unrelated microbial lineages can fulfill similar ecological and metabolic roles $[9,10]$. Therefore, different assemblages of bacterial phylotypes within hosts can be functionally convergent even in the absence of taxonomic congruence [11, 12]. For example, three distantly related species of insect-feeding bats from Africa were found to have host-specific assemblages of bacteria, but the predicted functional profiles of the three species' gut communities were largely convergent [13], supporting the observation that similar gut microbiome functions can be fulfilled by different sets of bacteria.

To better understand how microbes have influenced the evolution of their vertebrate hosts, it is essential to understand the functions they provide, as these functions may ultimately become targets of selection. If we consider microbes as aggregates of genes and traits, we might expect ecological filtering to operate more strongly at the level of microbial functions than species identity. We might also expect that nutritionally relevant functions should differ among hosts of different dietary habits, as transitions to new food resources would favor the retention of microbes capable of metabolizing novel food items. It is known that even subtle changes in diet within an animal's lifetime (e.g., as a result of habitat conversion) are associated with decreased functional capacity in the gut microbiome in primates [14]. Therefore, over evolutionary time, functional repertoires may diverge among hosts with different diets, although this may not unilaterally be the case in host clades that have more depauperate gut communities [12].

To test the hypothesis that microbiome functions should vary among mammals with different diets, we focused on bats as a model system. Bats, the second-most speciose order of mammals, are an ideal system in which to examine functional enrichment among hosts with different diets [15]. Unlike other well-studied host-microbe systems (e.g., primates [16-18] and rodents [19, 20]), the order Chiroptera contains independent dietary radiations into every known terrestrial feeding niche, but especially frugivory, nectarivory, and carnivory [21]. Within this phylogenetic context, it is therefore possible to analyze the enrichment of functional pathways in groups of species that have undergone independent transitions to similar diets. For example, transitions to frugivory occurred in two bat families, the Phyllostomidae and Pteropodidae, independent radiations that happened over millions of years of geographic isolation [22, 23]. Because both of these clades independently switched to a frugivorous lifestyle, it is possible to isolate the influence of host diet away from that of shared evolutionary history in structuring microbiome functions.

To test for enrichment of predicted functional microbial pathways among hosts with different feeding niches, we examined the gut microbiomes of 60 species spanning the full dietary diversity of bats, including insectivorous, frugivorous, omnivorous, sanguivorous (i.e., blood-feeding) and carnivorous species. Using $16 \mathrm{~S}$ rRNA gene profiling and phylogenetically-informed prediction of bacterial metabolic pathways, we functionally categorized more than 500 individual bat microbiomes and tested for differential enrichment of bacterial metabolic pathways across the five feeding niches. We used both multiple regression of matrices (MRM) and Random Forest decision trees to test the power of microbiome functions to predict host diet and host taxonomic identity.

Finally, we assessed how the enrichment of these inferred pathways is patterned across the bat phylogeny to understand whether they might be visible to natural selection, and thus inform or respond to the evolution of bat hosts. Considering inferred functional pathways as "traits" of the host, we tested this hypothesis by fitting our data to comparative models of trait evolution. Using a host phylogeny, trait values at the tips of the tree are used to compute values at ancestral nodes. Next, expected trait values are simulated under observed models of evolution. Finally, observed trait data can be compared with the trait values expected under each model, and model fit can be assessed using Akaike's Information Criterion (AIC) [24]. In particular, we were interested in determining if microbiome functional pathways evolve neutrally or in patterns that might invoke natural selection. To test this, we fit our observed functional pathways to four evolutionary trait models: White Noise, Brownian Motion, Early Burst, and Ornstein-Uhlenbeck. These models describe different evolutionary hypotheses about how continuous traits evolve along a phylogeny. The White Noise model assumes traits evolve as random draws from a common distribution, while Brownian Motion (BM) is a neutral 


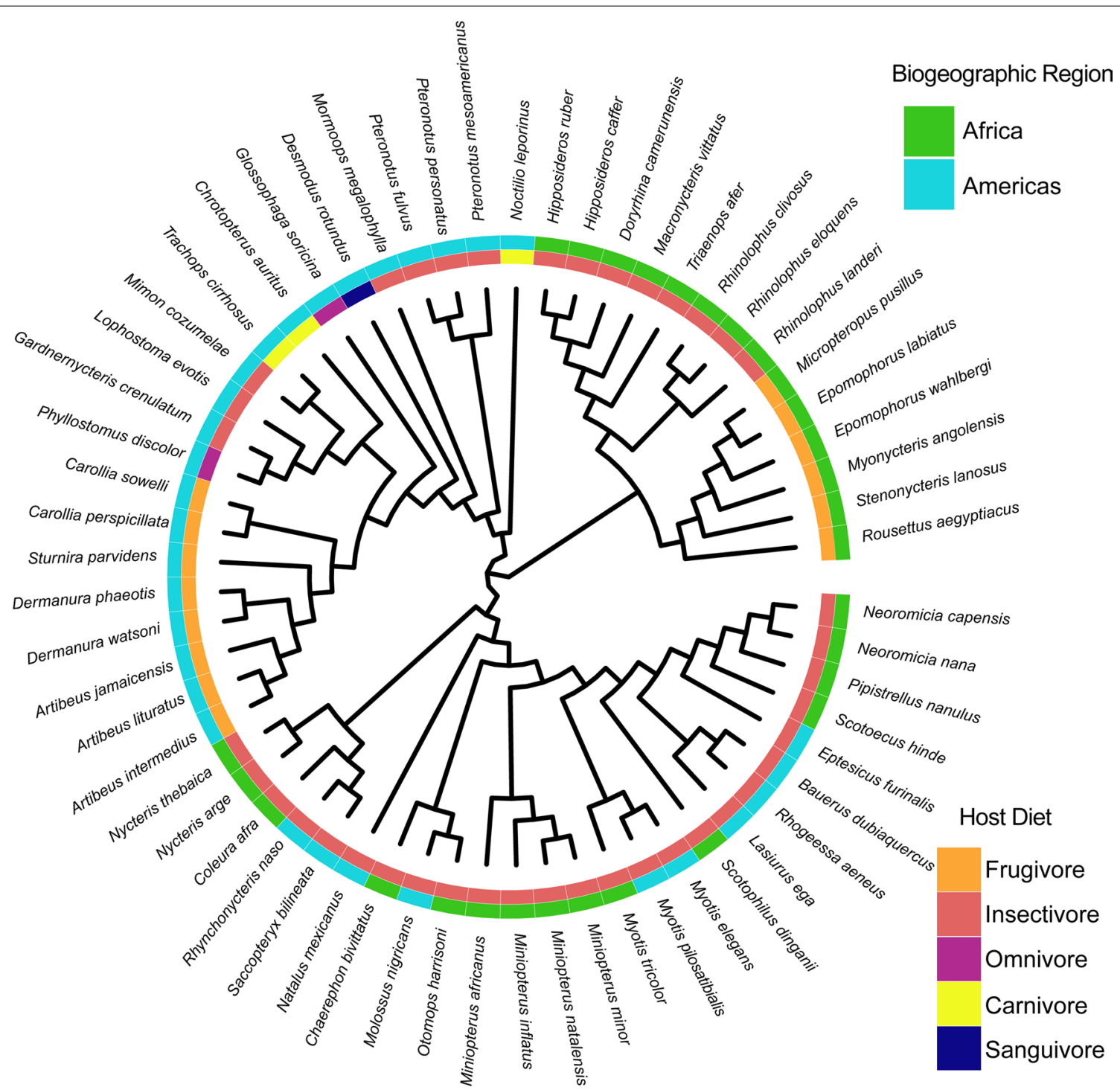

Fig. 1 Phylogenetic relationships between hosts sampled in this study. Pruned phylogeny was recovered from VertLife.org (Upham et al. 2019). Biogeographic origin of hosts is indicated in the outermost ring of tiles, while host feeding niche is indicated by the innermost ring of tiles

model often described as reflecting random drift as a result of mutation as hosts diverge through time. Early Burst (EB) models describe a trait that diversifies rapidly early on in host evolution and slows over time as niches are filled. Finally, the Ornstein-Uhlenbeck (OU) model, like Brownian Motion, is a random walk with an additional parameter that "pulls" traits toward some adaptive optimum, and thus, invokes selection [25].

\section{Results}

The full dataset contained 545 microbiome samples from representatives of 13 families of bats (42 genera, 60 species). This dataset samples all known bat feeding niches and includes instances of repeated independent dietary transitions to frugivory/nectarivory across the order (Fig. 1). Functional prediction with PICRUSt2 resulted in a feature table of 448 MetaCyc pathways [63]. Using PERMANOVA, we found that overall, predicted functional consortia were significantly differentiated by host taxonomy and diet, and that this was true regardless of whether we classified diet using a coarse or fine classification scheme (Fig. 2A, B, Additional file 1: Fig. S1; Table 1). Host taxonomy explained a greater percentage of the variation than diet, although both were significant factors (Table 1). For the pairwise tests, we found that predicted microbiome functions of frugivorous bats were 

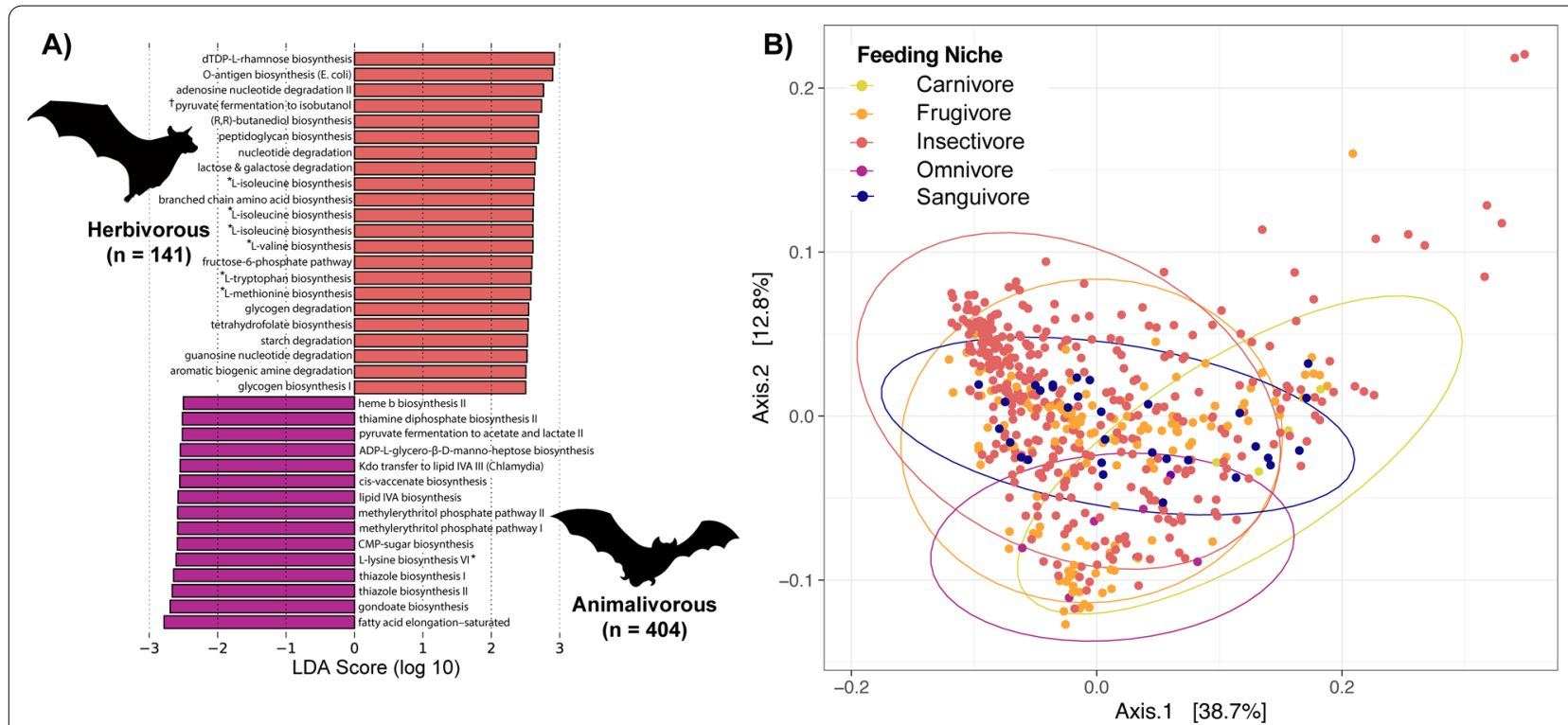

C)
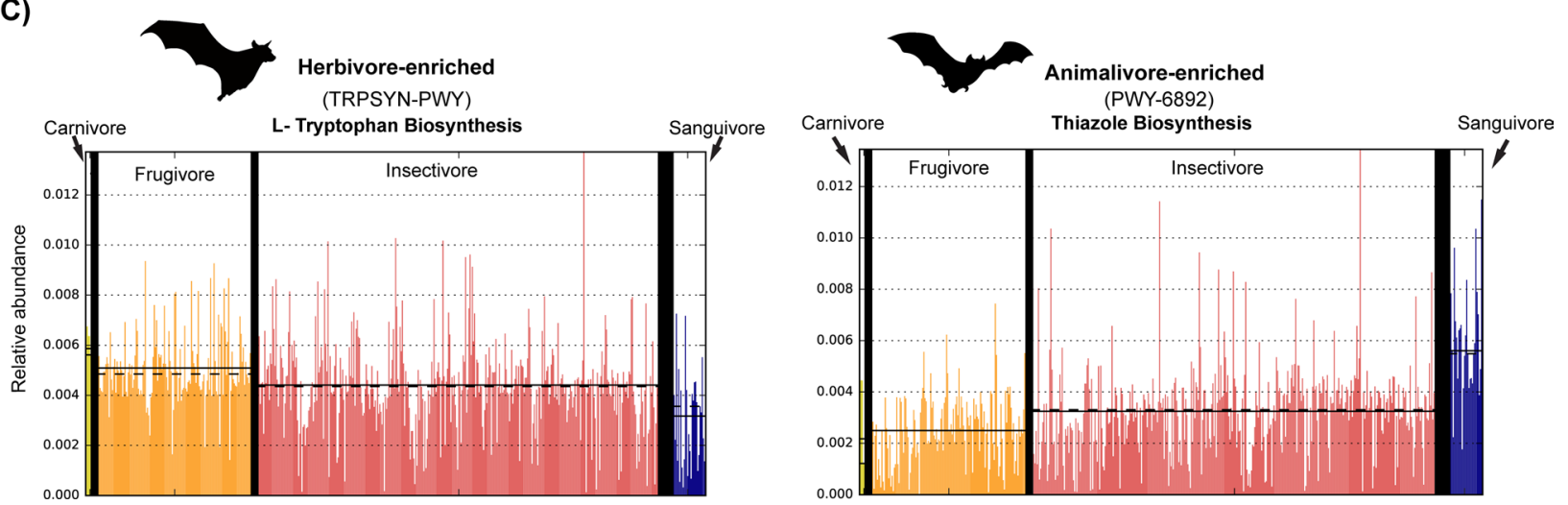

Fig. 2 Microbiome functions are differentially enriched across herbivorous and animalivorous bats. A Results of LDA-LEfSe analysis of metagenome functions between primarily herbivorous and animalivorous bats (cutoff LDA score $\geq 2.5$ ). The symbol + indicates an engineered pathway, while * indicates a pathway associated with synthesis of an essential amino acid. B Principal coordinates analysis of bat metagenome functions, where each dot represents an individual animal's metagenome. $\mathbf{C}$ Relative abundance of two functions determined to be differentially enriched in bats of different feeding guilds, where each bar represents one sample. Horizontal lines indicate mean relative abundance within groups. Omnivores are not depicted due to small sample size

significantly different from those of insectivores, carnivores, and sanguivores, but not different from omnivores (Table 2). Carnivorous bats were highly distinguishable from all other feeding guilds. Omnivorous bats overlapped with frugivorous and sanguivorous bats (Fig. 2B; Table 2) but were distinguishable from strict insectivores and carnivores. The ecologically hyper-specialized vampire bats (sanguivores) had distinctive predicted functional repertoires compared to all other feeding guilds except omnivores (Table 2).

When we examined which predicted pathways defined each of the dietary niches, LEfSe analysis showed that a total of 37 functional pathways were differentially abundant between primarily animal-feeding and
Table 1 Result of PERMANOVA of fine and coarse feeding niche on predicted microbiome functions

\begin{tabular}{lrccll}
\hline & Df & Sums of Sq & $\mathbf{F}_{\text {model }}$ & $\mathbf{R}^{\mathbf{2}}$ & $\boldsymbol{P}_{\text {adj }}$ \\
\hline Fine Niche & 5 & 0.65 & 8.67 & 0.06 & ${ }^{* *} 0.001$ \\
Host species & 55 & 2.49 & 3.02 & 0.24 & ${ }^{* *} 0.001$ \\
Residuals & 484 & 7.26 & - & 0.70 & \\
Total & 544 & 10.40 & - & 1.00 & \\
Coarse Niche & 2 & 0.28 & 9.28 & 0.03 & ${ }^{* *} 0.001$ \\
Host species & 58 & 2.87 & 3.29 & 0.27 & ${ }^{* *} 0.001$ \\
Residuals & 484 & 7.26 & - & 0.70 & \\
Total & 544 & 10.40 & - & 1.00 & \\
\hline
\end{tabular}

** are significant at the $P<0.05$ level after Benjamini-Hochberg correction 
Table 2 Pairwise PERMANOVA results for predicted functions among fine-scale feeding niches

\begin{tabular}{|c|c|c|c|c|c|}
\hline & $\begin{array}{l}\text { Sums } \\
\text { of Sq }\end{array}$ & $\mathrm{F}_{\text {model }}$ & $R^{2}$ & P-value & $P_{\text {adj }}$ \\
\hline Carnivore $\leftrightarrow$ Frugivore & 0.103 & 6.913 & 0.046 & 0.001 & $* * 0.004$ \\
\hline$\leftrightarrow$ Insectivore & 0.114 & 5.779 & 0.016 & 0.006 & $* * 0.013$ \\
\hline$\leftrightarrow$ Omnivore & 0.060 & 3.791 & 0.275 & 0.008 & ${ }^{* *} 0.015$ \\
\hline$\leftrightarrow$ Sanguivore & 0.079 & 5.014 & 0.125 & 0.003 & ${ }^{* *} 0.009$ \\
\hline Frugivore $\leftrightarrow$ Insectivore & 0.234 & 12.740 & 0.025 & 0.001 & ${ }^{* *} 0.004$ \\
\hline$\leftrightarrow$ Omnivore & 0.035 & 2.413 & 0.016 & 0.041 & 0.061 \\
\hline$\leftrightarrow$ Sanguivore & 0.246 & 16.856 & 0.090 & 0.001 & $* * 0.004$ \\
\hline Insectivore $\leftrightarrow$ Omnivore & 0.058 & 2.961 & 0.008 & 0.028 & ${ }^{* *} 0.047$ \\
\hline$\leftrightarrow$ Sanguivore & 0.249 & 12.844 & 0.032 & 0.001 & ${ }^{* *} 0.004$ \\
\hline Omnivore $\leftrightarrow$ Sanguivore & 0.010 & 1.203 & 0.194 & 0.440 & 0.507 \\
\hline
\end{tabular}

Comparisons indicated with ** are significant at the $P<0.05$ level after Benjamini-Hochberg correction

plant-feeding bats (Fig. 2A, Table 3). All of the enriched pathways in animalivorous bats were associated with biosynthesis (93.7\%) or generation of precursor metabolites $(6.3 \%)$, while pathways enriched in herbivorous bats were split between biosynthesis (62.0\%), degradation, utilization, and assimilation functions (33.3\%), and generation of precursor metabolites (4.7\%). Six of the pathways enriched in herbivorous bats were associated with proteinogenic amino acid biosynthesis, specifically the production of the essential amino acids isoleucine, valine, tryptophan, and methionine [64]. Figure $2 \mathrm{C}$ (left) depicts the differential abundance of one of these pathways, the L-tryptophan biosynthesis pathway, among feeding niches. Pathways enriched in animal-feeding bats were more general, and were split among fatty acid, amino acid, and secondary metabolite processing pathways (Fig. 2A, Table 3). The relative abundance of one animalivore-enriched pathway, the thiazole biosynthesis pathway, is shown in Fig. 2C (right). Another pathway found to be enriched in frugivores was determined to be PWY-7111, an engineered pathway not known to occur naturally in any bacterial species. The contributing bacterial ASV for this pathway likely did not match closely to a known reference microorganism during the PICRUSt2 predictions (Fig. 2A; Table 3). We also performed LEfSe differential abundance analysis on the fine-scale niche groupings. The results were consistent with the coarse analysis, with some additional pathways contributing to the observed differences among dietary ecologies (e.g., ketogluconate metabolism for frugivores, cobinamide salvage in sanguivores) (Fig. 3).

Matrix regressions (MRM) run on the full dataset determined that only the patristic distance (i.e., phylogenetic distance between hosts) was predictive of microbiome functional dissimilarity (MRM $\left.P_{\text {phylo }}=0.01\right)$ while ecological distances computed from EltonTraits quantitative data were not significantly predictive (MRM $P_{\text {ecol }}=0.38$ ). However, matrix regression analysis requires merging all within-species replicates to create a distance matrix based on averaged values for each species. To account for high amounts of inter-individual variation in microbiomes, we also subjected the data to Random Forest analysis to test the predictive power of metagenome functions on an individual sample, rather than wholespecies, basis.

Random Forest (RF) analyses were conducted to test the ability of metagenomic functions to classify bats into dietary guilds as well as host family and genus. For the coarse (animalivorous vs. herbivorous) niche classification model, the out-of-bag error rate was $13.2 \%$. Within-class error varied according to host niche; the model performed particularly well at identifying primarily animalivorous bats based on metagenome functions, but less so for primarily plant-feeding animals, and very poorly for omnivores (Table 4). The fine niche model performed slightly worse, with an OOB of $15.6 \%$. Similarly, the model performed best at predicting the insectivorous classifications, followed by frugivorous, and struggled substantially to predict omnivores, carnivores, and sanguivores (Table 5 ). Models for predicting host family and genus performed poorly, with out-of-bag error rates of $49.4 \%$ and $58.4 \%$, respectively (Additional file 4 : Table S3, Additional file 5: S4). We tested the accuracy of the RF niche models using leave-one-out cross-validation, a resampling procedure used to estimate how a model is expected to perform in general when used to make predictions on data not used during the training of the model. Cross-validation on 500 trees produced an accuracy rate of $86.6 \%(\mathrm{Kappa}=0.626)$ for the coarse classification model and $84.2 \%(\mathrm{Kappa}=0.650)$ for the fine classification model. We next sorted the functional variables by mean decrease in model accuracy (i.e. variable importance in training the model). The resulting top ten most informative features for classifying host diets are shown in Fig. 4.

Phylogenetic comparative analyses were performed on the ten most informative functional pathways identified by random forest analysis (Fig. 4). Our sampling of the clade encompassed 13 families, representing about $60 \%$ of the family-level diversity of extant bats [65]. The enrichment or depletion of predicted microbiome pathways was not distributed randomly with respect to host phylogeny; for example, the saturated fatty acid elongation pathway was selectively underenriched in the Pteropodidae and Phyllostomidae (Fig. 5A). To test for phylogenetic signal in pathway enrichment, we calculated Pagel's $\lambda$ for all ten metagenomic pathways. All pathways had low phylogenetic signal in general, with P164-PWY 
Table 3 Differentially enriched metagenome functions recovered from LEfSe analysis

\begin{tabular}{|c|c|c|c|c|}
\hline MetaCyc Pathway & Superpathway & Enrichment & LDA Score & Wilcoxon $P$ \\
\hline DTDPHRAMSYN_PWY & Carbohydrate Biosynthesis & Herbivorous & 2.92 & $1.27 \mathrm{E}-14$ \\
\hline OANTIGEN-PWY & Carbohydrate Biosynthesis & Herbivorous & 2.90 & $8.91 \mathrm{E}-15$ \\
\hline SALVADEHYPOX-PWY & Nucleoside and Nucleotide Degradation & Herbivorous & 2.76 & $2.10 \mathrm{E}-07$ \\
\hline tPWY-7111 & Engineered & Herbivorous & 2.74 & $6.98 \mathrm{E}-09$ \\
\hline P125-PWY & Other Biosynthesis & Herbivorous & 2.69 & $7.95 \mathrm{E}-20$ \\
\hline PWY-6471 & Cell Structure Biosynthesis & Herbivorous & 2.69 & $8.12 \mathrm{E}-07$ \\
\hline PWY-6353 & Nucleoside and Nucleotide Degradation & Herbivorous & 2.66 & $6.18 \mathrm{E}-07$ \\
\hline LACTOSECAT-PWY & Carbohydrate Degradation & Herbivorous & 2.64 & $1.40 \mathrm{E}-13$ \\
\hline *PWY-5101 & Amino Acid Biosynthesis & Herbivorous & 2.63 & $1.10 E-07$ \\
\hline BRANCHED-CHAIN-AA-SYN-PWY & Amino Acid Biosynthesis & Herbivorous & 2.62 & $3.68 \mathrm{E}-09$ \\
\hline *PWY-5103 & Amino Acid Biosynthesis & Herbivorous & 2.61 & $2.09 \mathrm{E}-09$ \\
\hline *ILEUSYN-PWY & Amino Acid Biosynthesis & Herbivorous & 2.61 & $8.36 \mathrm{E}-08$ \\
\hline *VALSYN-PWY & Amino Acid Biosynthesis & Herbivorous & 2.61 & $8.36 \mathrm{E}-08$ \\
\hline P124-PWY & Fermentation & Herbivorous & 2.59 & $3.39 E-09$ \\
\hline *TRPSYN-PWY & Amino Acid Biosynthesis & Herbivorous & 2.58 & 7.93E-07 \\
\hline *HSERMETANA-PWY & Amino Acid Biosynthesis & Herbivorous & 2.58 & $1.23 \mathrm{E}-06$ \\
\hline GLYCOCAT-PWY & Polymeric Compound Degradation & Herbivorous & 2.55 & 7.96E-06 \\
\hline PWY-6612 & Cofactor, Prosthetic Group, Electron Carrier, and Vitamin Biosynthesis & Herbivorous & 2.54 & $7.51 \mathrm{E}-09$ \\
\hline PWY-6737 & Polymeric Compound Degradation & Herbivorous & 2.53 & $2.33 \mathrm{E}-05$ \\
\hline PWY-6608 & Nucleoside and Nucleotide Degradation & Herbivorous & 2.52 & $8.16 \mathrm{E}-05$ \\
\hline PWY-7431 & Amine and Polyamine Degradation & Herbivorous & 2.50 & $3.29 \mathrm{E}-08$ \\
\hline GLYCOGENSYNTH-PWY & Carbohydrate Biosynthesis & Herbivorous & 2.50 & $1.83 \mathrm{E}-05$ \\
\hline HEMESYN-PWY & Cofactor, Prosthetic Group, Electron Carrier, and Vitamin Biosynthesis & Animalivorous & 2.50 & 0.0165 \\
\hline PWY-6895 & Cofactor, Prosthetic Group, Electron Carrier, and Vitamin Biosynthesis & Animalivorous & 2.51 & $9.74 \mathrm{E}-09$ \\
\hline PWY-5100 & Fermentation & Animalivorous & 2.52 & 0.013 \\
\hline PWY0-1241 & Carbohydrate Biosynthesis & Animalivorous & 2.55 & $1.68 \mathrm{E}-06$ \\
\hline PWY-6467 & Cell Structure Biosynthesis & Animalivorous & 2.55 & 0.00052 \\
\hline PWY-5973 & Fatty Acid and Lipid Biosynthesis & Animalivorous & 2.55 & $1.88 \mathrm{E}-05$ \\
\hline NAGLIPASYN-PWY & Cell Structure Biosynthesis & Animalivorous & 2.58 & $6.92 \mathrm{E}-05$ \\
\hline PWY-7560 & Secondary Metabolite Biosynthesis & Animalivorous & 2.58 & 0.00044074 \\
\hline NONMEVIPP-PWY & Secondary Metabolite Biosynthesis & Animalivorous & 2.58 & 0.0004 \\
\hline PWY-1269 & Carbohydrate Biosynthesis & Animalivorous & 2.59 & $5.64 \mathrm{E}-05$ \\
\hline *PWY-5097 & Amino Acid Biosynthesis & Animalivorous & 2.61 & 0.0004 \\
\hline PWY-6892 & Cofactor, Prosthetic Group, Electron Carrier, and Vitamin Biosynthesis & Animalivorous & 2.64 & $3.33 \mathrm{E}-08$ \\
\hline PWY-6891 & Cofactor, Prosthetic Group, Electron Carrier, and Vitamin Biosynthesis & Animalivorous & 2.66 & $1.09 \mathrm{E}-09$ \\
\hline PWY-7663 & Fatty Acid and Lipid Biosynthesis & Animalivorous & 2.70 & $2.92 \mathrm{E}-09$ \\
\hline FASYN-ELONG-PWY & Fatty Acid and Lipid Biosynthesis & Animalivorous & 2.78 & $3.86 \mathrm{E}-09$ \\
\hline
\end{tabular}

All LDA scores were retained only where LDA $\geq 2.5$ and are shown rounded to the second decimal place. Wilcoxon test was considered to be significant if $P \leq 0.05$. + is an engineered metabolic pathway, while * notes a synthesis pathway for an essential or conditionally essential amino acid

(purine nucleobase degradation) having $\lambda$ statistically equivalent to zero. The pathways OANTIGEN-PWY (O-antigen biosynthesis pathway) and BRANCHEDCHAIN-AA-SYN-PWY (branched chain amino acid synthesis superpathway) both had low phylogenetic signal with $\lambda=0.1$. The pathways PWY-6612 (tetrahydrofolate biosynthesis superpathway), LACTOSECAT-PWY (lactose and galactose degradation superpathway), and DTDPRHAMSYN-PWY (dTDP- $\beta$-L-rhamnose biosynthesis) all had $\lambda=0.12$. The pathways with the highest phylogenetic signal were the FASYN-ELONG-PWY (saturated fatty acid elongation, $\lambda=0.13)$, P125-PWY ( $R, R)$-butanediol biosynthesis, $\lambda=0.16$ ), and PWY-1269 (CMP3-deoxy-D-manno-octulosonate biosynthesis, $\lambda=0.19$ ) respectively (Fig. 5B).

In terms of model fit, all AIC weight was split between the OU and White Noise models, with Brownian Motion and Early Burst models receiving none of the AIC weight 


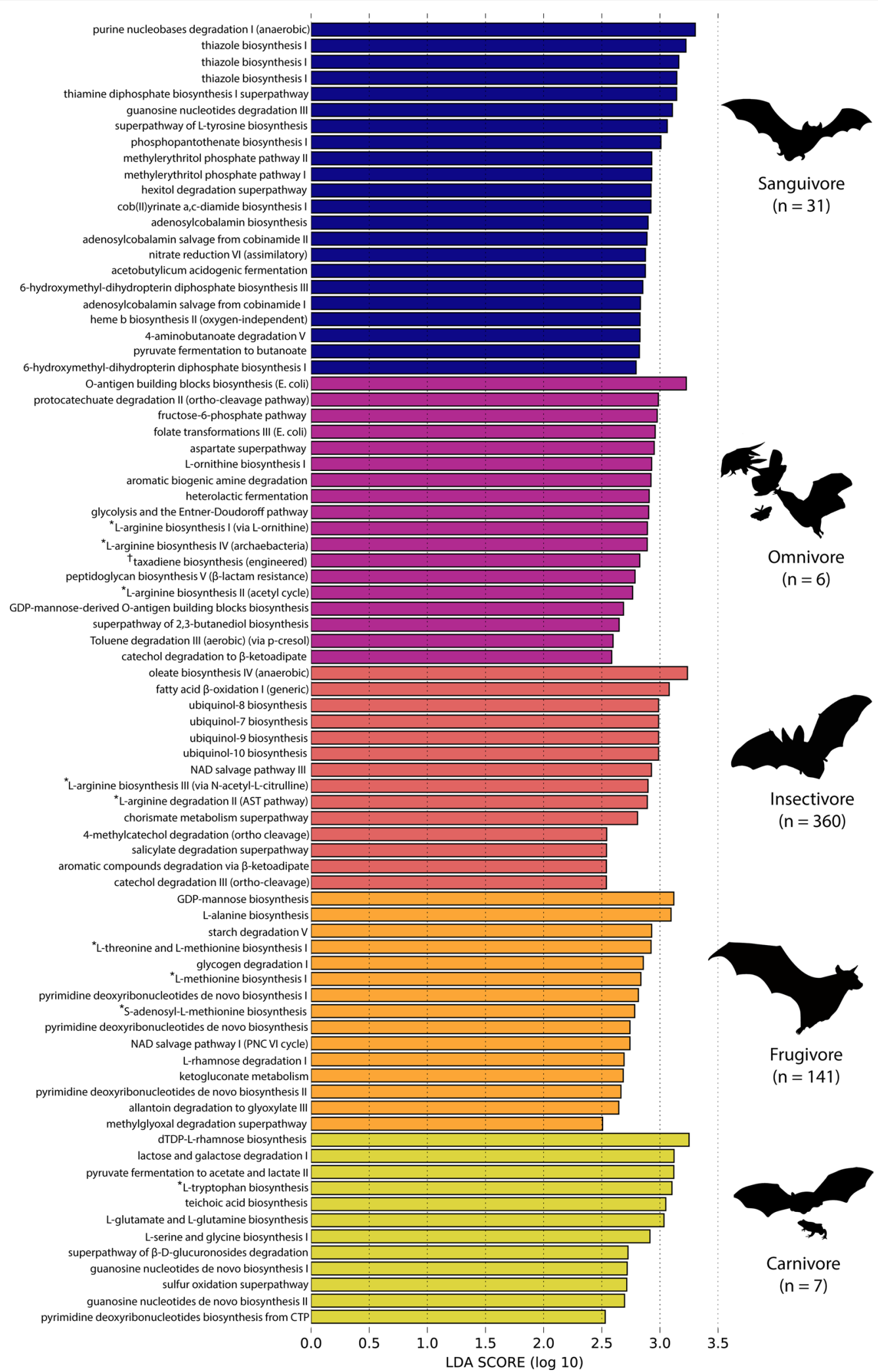

Fig. 3 Functional pathways differentially enriched among dietary guilds. LEfSe results for fine scale niche with minimum LDA score cutoff of $\geq 2.5$ 
Table 4 Confusion matrix for the coarse niche random forest model

\begin{tabular}{lclc}
\hline & Animalivorous & Omnivorous & Herbivorous \\
\hline Animalivorous & 386 & 0 & 12 \\
Omnivorous & 2 & 0 & 4 \\
Herbivorous & 54 & 0 & 87 \\
\hline
\end{tabular}

Within-class error rates were $3.0 \%$ for Animalivores, $100 \%$ for omnivores, and $38 \%$ for herbivores

Table 5 Confusion matrix for fine-scale niche random forest model

\begin{tabular}{lccccc}
\hline & Carnivore & Frugivore & Insectivore & Omnivore & Sanguivore \\
\hline $\begin{array}{l}\text { Carni- } \\
\text { vore }\end{array}$ & 0 & 0 & 6 & 0 & 0 \\
$\begin{array}{l}\text { Frugi- } \\
\text { vore }\end{array}$ & 92 & 49 & 0 & 0 \\
$\begin{array}{l}\text { Insec- } \\
\text { tivore }\end{array}$ & 0 & 14 & 346 & 0 & 0 \\
$\begin{array}{l}\text { Omni- } \\
\text { vore }\end{array}$ & 4 & 2 & 0 & 0 \\
$\begin{array}{l}\text { San- } \\
\text { guiv- } \\
\text { ore }\end{array}$ & 1 & 8 & 0 & 22 \\
\hline
\end{tabular}

Within-class error rates were $100 \%$ for carnivores, $34.8 \%$ for frugivores, $3.9 \%$ for insectivores, $100 \%$ for omnivores, and $29 \%$ for sanguivores

for any pathway. The White Noise model received $>50 \%$ of the weight for 5 of the 10 pathways tested, with another 2 models sharing 50-50 split between White Noise and OU models. The OU model received $>50 \%$ of the weight for only three pathways- saturated fatty acid elongation, tetrahydrofolate biosynthesis and salvage, and lactose and galactose degradation (Fig. 5B).

\section{Discussion}

Our current understanding of host-microbe interactions in wild mammals is mostly limited to observations of phylosymbiosis between host clades and their microbiotas. While these tests are a necessary and foundational step in symbiosis research, the true impacts of microbial symbionts on host fitness and evolution cannot be fully understood without more explicit inventories of the functions that these communities contribute to their hosts. In this study, we found that bats with different dietary specializations have microbiomes enriched with potentially adaptive predicted functions (Table 3), and that these functions can be used to predict the dietary classification of the host (Tables 4,5). When we considered bats as either primarily herbivorous or animalivorous, very few predicted functions could significantly discriminate among the groups. However, of the pathways that were found to be enriched in herbivorous (i.e. fruit- or nectar-feeding) bats, several were pathways associated with the production of the essential amino acids methionine, valine, isoleucine, and tryptophan (Fig. 2A; Table 3). Essential amino acids are those than cannot be synthesized de novo by the host; they must either already be present in the diet or produced through microbial metabolism and absorbed through the host intestine $[26,27]$. Essential amino acids may be particularly limiting nutrients for obligate frugivores; fruits consumed by fruit bats are deficient in protein compared with insects $[28,29]$, such that existing on a diet primarily consisting of fruit may pose nutritional challenges that can be partially overcome by the metabolic contributions of symbiotic microbes. In a mouse model, it was shown that gut microbes can provision essential amino acids when hosts are fed on protein-deficient diets [30], lending further evidence that gut bacteria play essential nutritional roles in their hosts.

While the exact fruits consumed by many frugivorous bat species are unknown, phyllostomid bats are known to feed on Piper and Ficus fruits, which are considered to be nutritionally poor food resources [28]. The bacterial genus Erwinia was prevalent in the microbiotas of Belizean fruit bats; members of this genus are known to provide supplemental protein to herbivorous adult olive flies, suggesting that they may fulfill a similar role in the microbiomes of fruit bats from Belize [31]. Fruit consumed by the pteropodid species Micropteropus pusillus and Epomops buettikoferi were shown to contain 3.3\% protein by dry mass, indicating that African fruit bats feed on nutritionally poor fruits as well [29]. In African fruit bats, the bacterial genus Gemella was enriched compared to insectivores [11]. This genus is predicted to be capable of synthesizing methionine according to the Kyoto Encyclopedia of Genes (KEGG), and may partially explain the enrichment in L-methionine pathways we observed in frugivores (Fig. 3). Other functions enriched in herbivorous bats were related to carbohydrate degradation (e.g., glycogen and starch), as well as biosynthesis of the B-vitamin folate. Enrichment in these bacterial pathways may be complementary to the nutritional composition of various fruits, which are made up primarily of water and simple carbohydrates with relatively few proteins, vitamins, and minerals [28, 32, 33].

Differential enrichment analysis detected more functional pathways discriminating among dietary groups when classified more finely (Fig. 3). Notably, in our sample of 23 vampire bat (Desmodus rotundus) microbiomes, we found many pathways related to cofactor and vitamin biosynthesis, inorganic nutrient metabolism, and amine degradation to be enriched (Fig. 3). This is consistent with previous findings by Zepeda-Mendoza et al. (2018), which showed enrichment of microbial genes related to 


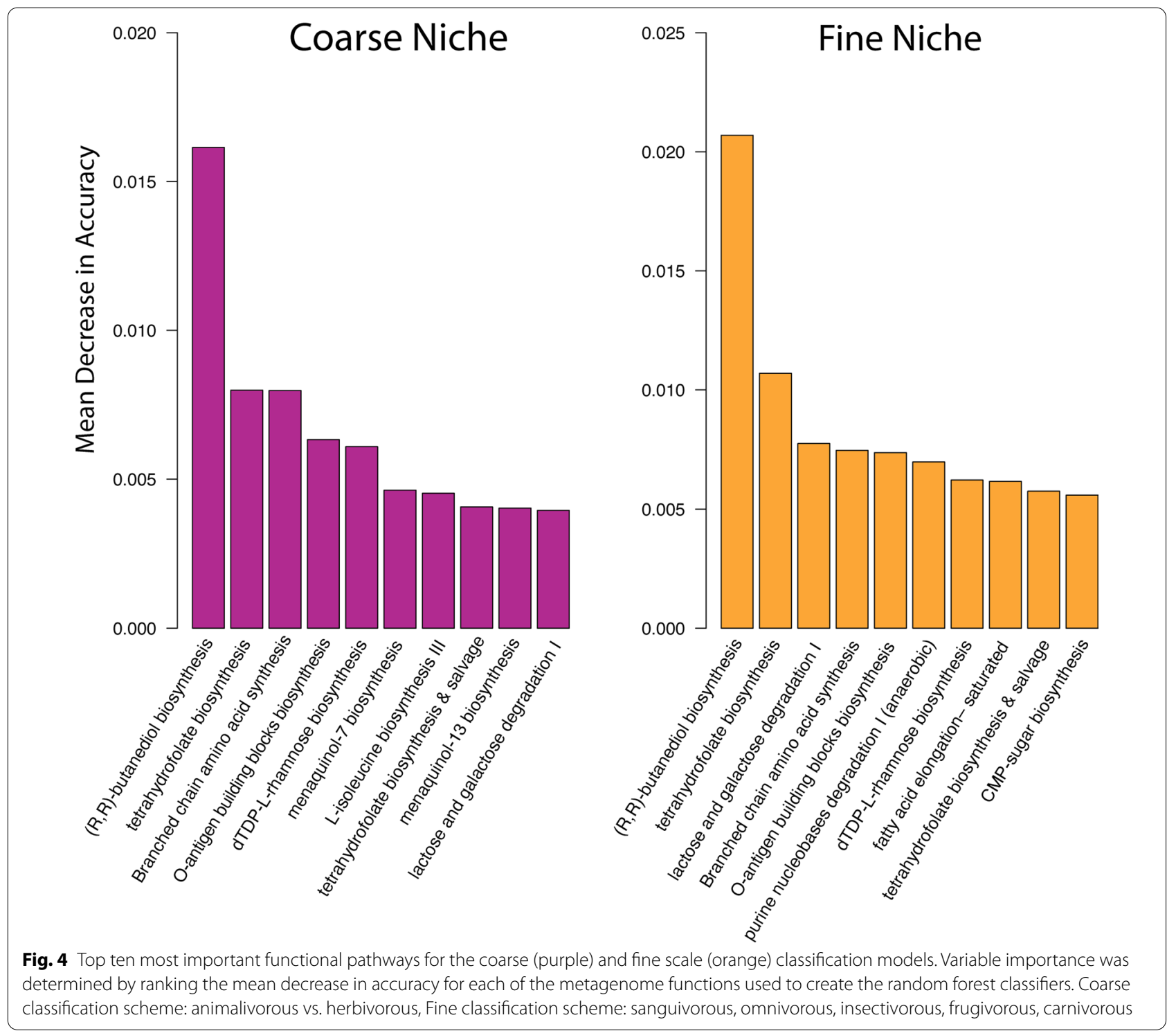

cofactor and vitamin metabolism, siderophore biosynthesis (important for handling iron and heme), and amino acid metabolism [2]. To link these functions to bacterial phylotypes present in the vampire bat microbiome, we find that vampire bat gut microbiotas are characterized by high relative abundance of Peptostreptococcaceae, which have the rare ability among microbes to ferment amino acids [34, 35]. Blood is $78 \%$ liquid, but its solid cellular phase contains $93 \%$ proteins and less than $1 \%$ carbohydrates [36], suggesting that members of this bacterial clade may assist in protein processing in the vampire bat host.

Overall, animalivorous bats had metagenomes that were characterized by vitamin, proteinogenic amino acid, fatty acid, and carbohydrate synthesis (Table 3). This more generalized suite of microbial functions is likely a byproduct of energetic demands on insectivorous hosts. Insect-eating bats rely on recently consumed exogenous resources to fuel flight, which may possibly select for microbes which can generate other, non-combustible metabolites for later use by the host $[37,38]$.

In addition to identifying specific pathways associated with the feeding habits of these species, we wanted to know how predictive overall functional composition was of dietary guild. Our random forest models performed well at predicting host diet, with accuracy rates between 80 and $85 \%$ regardless of whether we classified diet using a coarse or fine classification scheme. The models were best at predicting insectivorous or primarily animalivorous species based on their gut microbiomes 


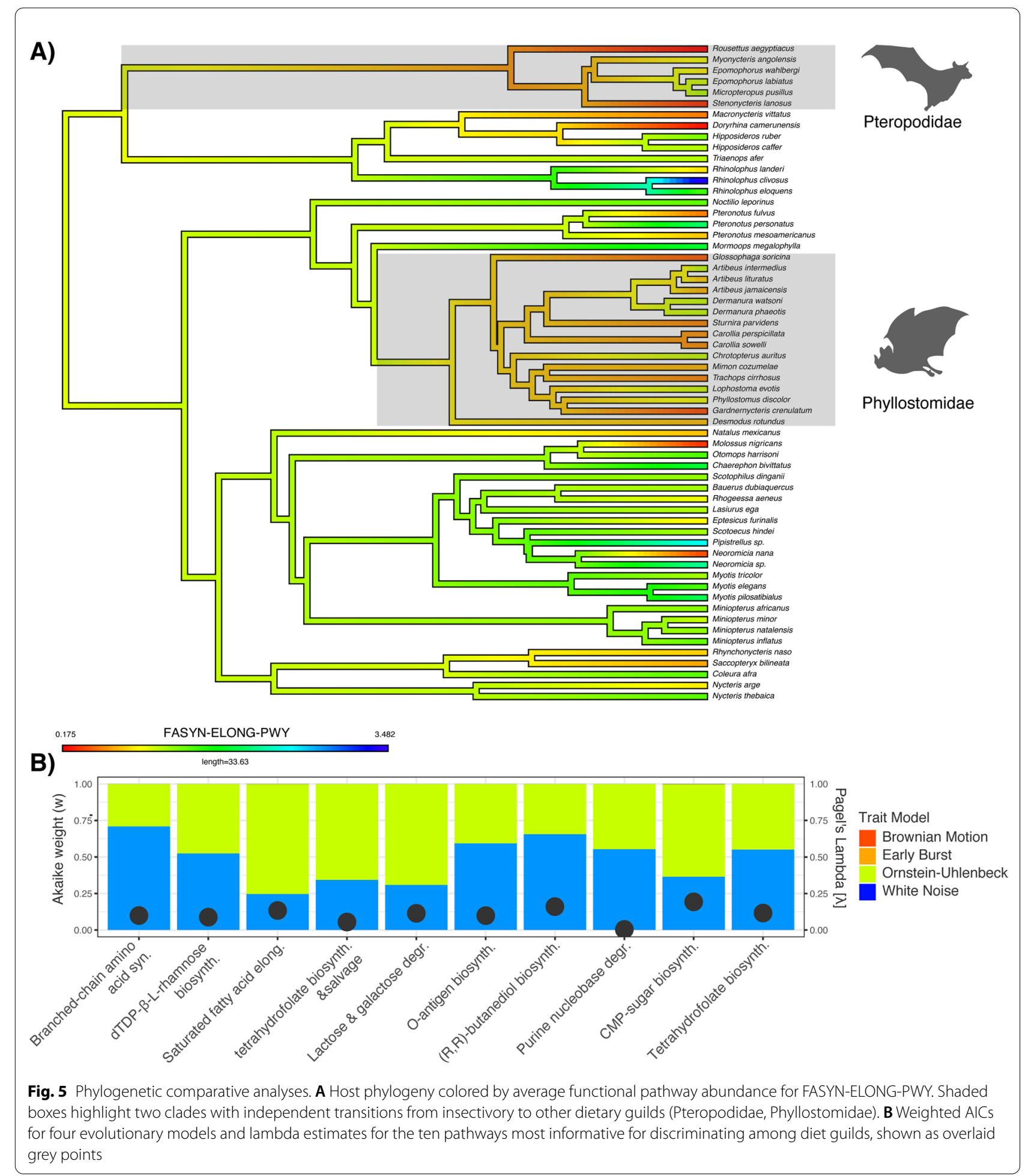

but were substantially worse at predicting frugivores and omnivores. It is important to note that many dietary specializations, including frugivory and nectarivory, are more labile than previously thought [39-41]. For instance, some species of bats are known to occasionally take insects despite being considered "frugivores" [42], so rather than existing as discrete, closed niches, many bat species probably fall along a spectrum running 
from primarily plant-feeding, to omnivorous, to primarily animal-feeding. In light of this view, it is unsurprising that the random forest models failed to correctly identify omnivores $100 \%$ of the time. Omnivorous microbiomes are not likely characterized by their own suites of functions per se. Rather, they are more likely functionally intermediate between strict insectivores and frugivores, which the PCoA of predicted metagenome functions supports (Fig. 2B). The matrix regressions did not detect a meaningful correlation between host metagenomic distances and diet; however, given the high level of withinspecies microbiome variation in bats $[11,12,43]$, it is likely that averaging functions within species so that they match the taxon-level dietary data from EltonTraits introduces inappropriate levels of noise to the distancebased analysis. Taken with our random forest results, we conclude that host diet and microbiome functions may be related on a per-sample rather than per-taxon basis. The phylogenetic matrix regression also recovered a relationship between host phylogeny and microbiome function, suggesting that overall functional profiles may be related to host evolutionary history. However, the MRM method requires collapsing all of the microbiome functional variation into patristic distances that can obscure more finescale patterns. Random Forest models were unable to predict host family or genus membership using microbiome functions alone (Additional file 4: Table S3, Additional file 5: Table S4), suggesting that while functions are characteristic of host diet, they are not able to discriminate among related hosts.

By contrast, the comparative phylogenetic analyses, which were performed on individual pathways rather than distances, detected very low phylogenetic signal in all of the tested pathways, with the data for most pathways best fitting a white noise, or phylogeny independent, model of trait evolution (Fig. 5B). However, three critical metabolic superpathways dealing with unsaturated fatty acid elongation, folate biosynthesis, and lactose catabolism were more heavily weighted toward an $\mathrm{OU}$ model of evolution (Fig. 5B). The OU model differs from a Brownian Motion model in that a stochastically varying trait is assumed to evolve toward an optimal value rather than neutrally along the phylogeny [44]. While we cannot say for certain whether the pathways fitting the selection-invoking evolutionary model are optimized to host ecology, when these pathways are mapped onto the host phylogeny, it is clear that their enrichment or depletion is mostly clustered in the clades that have experienced independent transitions away from insectivory, the Phyllostomidae and the Pteropodidae (Fig. 5A). Taken together with the results of the differential enrichment analyses, we hypothesize that a subset of metagenome functions may respond to selective pressures imposed by host diet, such that hosts with nutritionally challenging diets favor the retention of microbial functions that help facilitate their metabolic needs. A major caveat of this approach is that microbiome functions need to be heritable to be considered as traits of the host. Current evidence for vertical transmission is lacking for mammals, but there is some evidence that wild animals have a higher proportion of heritable gut bacteria than previously thought [45]. Given that the gastrointestinal traits governing this filter have a genetic, and therefore potentially heritable, basis [46], we also suggest that some microbiome members may be considered as "functionally inherited" if microbial metabolites, rather than species, are the actual targets of host selection [47].

While our data suggests a role for microbes in host dietary evolution, our results come with some important limitations. One consideration is that microbiomes can shift in response to seasonal variation in diet which would not be captured by our cross-sectional sampling scheme. In addition, although PICRUSt2 has been shown to perform well at predicting functional pathways, this algorithm fundamentally relies on the completeness of microbial gene databases that link pathways with bacterial species. Because the bat microbiome is relatively poorly characterized, some taxa may not have particularly close matches in the database. In addition, amplicon-based functional predictions assume that all pathways are active. Direct inventories with shotgun metagenomic methods can be applied in the future to address database completion issues, while metatranscriptomics can address which members of the community are actively transcribing vs. dead or dormant (e.g., [48]). Metabolomic and isotopic techniques can further help to pinpoint specific metabolites contributed by the host's own physiological process versus those created by microbes [30, 49].

Our results suggest that bats across various feeding niches may rely on their gut symbionts to fulfill essential metabolic roles that are related to host dietary ecology, though the strength of this dependence likely depends on the level of host dietary specialization. These results recontextualize our understanding of host-microbe interactions within bats. Two recent studies did not detect a signal of phylosymbiosis among bats and their gut microbiotas, perhaps suggesting that it is unlikely that bats depend on their microbiomes as much as other vertebrates because the energetic demands of flight make maintaining these associations too costly [11, 12]. Our results demonstrate that numerous bacterial pathwayswhich may be encoded by a taxonomically diverse set of organisms-are correlated with dietary specialization in bats, suggesting at least some role for microbes in their ecological diversification. Questions still remain 
regarding the strength of this association compared with more obligately associated partners (e.g. cattle and their ruminal bacteria, insects with obligate endosymbionts). While our data cannot speak to the strength of association between bats and their bacteria, we suggest that selection on the microbiome may act more at the level of metabolic functions than on bacterial taxonomy. This interpretation may help to explain why bats have such high inter-individual variation in microbiome taxonomic composition. In addition, bat longevity may play a role in generating such variation. Bats are incredibly long lived for their body sizes [50], which may allow them to more thoroughly sample their environment for microbes throughout the course of their lifetimes. This hypothesis would help explain why bats show strong geographic patterning in microbiome taxonomic composition [51]. Few studies explicitly track individual bat microbiome turnover through time, and field studies on age-related phenomena in bats are limited due to the logistical challenges of recapturing individuals throughout their long adult life stages. Longitudinal studies of the microbiomes of laboratory-kept individuals could potentially test hypotheses about the stability of these relationships through time and with changing diets, adding valuable insight to the dynamics of bat-microbiome symbioses.

\section{Conclusions}

Our study found differential enrichment in predicted bacterial metabolic pathways-including essential amino acid synthesis, fatty acid biosynthesis, and the generation of cofactors and vitamins essential for proper nutrition-across bat dietary guilds. These results represent novel insights into potential metabolic collaborations between gut microbes and their wild mammalian hosts. Future studies can add further depth and resolution to the patterns we identified here by including more direct functional inference methodologies, such as shotgun metagenomics and metatranscriptomics. In addition, experimental approaches using metabolomic tools can be used to further partition the nutrient landscape of mammals between endogenously synthesized products and those provisioned by symbiotic gut bacteria. Our results, which cover a large proportion of extant bat diversity, provide novel functional insights into an ecologically and evolutionarily rich host-microbe system.

\section{Methods}

\section{Data collection}

For this meta-analysis, we combined three bat microbiome data sets, two of which were previously published and one that was generated as part of this study (Additional file 3: Table S2). The $16 \mathrm{~S}$ rRNA gene data for African bats were downloaded from the QIITA database from a study conducted by Lutz et al. 2019 [11]. This dataset contained 402 guano samples (31 species), and was prepared according to the Earth Microbiome Project protocols targeting the V4 region of the $16 \mathrm{~S}$ rRNA gene (515f/806r) [52]. We also included previously published vampire bat microbiotas from Ingala et al. (2019) ( $n=23,1$ species) to increase ecological coverage, which also included $16 \mathrm{~S}$ genes sequenced using the $\mathrm{V} 4$ region (515f/806r) [25].

New data were generated from fecal samples of bat species from the Americas captured in and around the Lamanai Archaeological Reserve in Orange Walk District, Belize $\left(17.75117^{\circ} \mathrm{N}, 88.65446^{\circ} \mathrm{W}\right)$ in April-May of 2016, 2017, and 2018 ( $n=114,28$ species). During field sampling, we adhered to the best practices for humane capture and handling of live mammals outlined by the American Society of Mammalogists [53], and all field protocols were approved by institutional animal care and use committees at the American Museum of Natural History (AMNH) IACUC-20180123 and Southern Connecticut State University (SCSU) IACUC S15-01.18. Briefly, bats were live captured in ground-level mist nets or harp traps and placed into individual clean cloth holding bags. Fecal samples were collected directly from bats or from the bottom of holding bats within $30 \mathrm{~min}$ of defecation using sterilized forceps. Each sample was placed into a sterile barcoded tube and immediately preserved in liquid nitrogen. Between uses, holding bags were washed in an industrial laundry to minimize cross-contamination of fecal samples, and forceps were twice sterilized between uses with a 10\% DNA-Away solution (Molecular Bioproducts, Inc., San Diego, CA) and water. Samples were shipped frozen to the $\mathrm{AMNH}$ and stored at $-80{ }^{\circ} \mathrm{C}$ prior to DNA extraction.

\section{Dietary classification scheme}

Because of the limited within-guild sample sizes for some dietary categories, such as carnivores, bats were classified into both "coarse" (Animalivorous or Herbivorous) and "fine" (Frugivorous, Carnivorous, Insectivorous, Sanguivorous, Omnivorous) dietary categories for statistical testing. This classification scheme was based on a thorough review of recent literature, taking into account newer diet studies that have overturned or expanded previous assumptions about host diet [e.g., 25, 26]. Still, many species do not fit neatly into dietary guilds because their feeding habits vary seasonally during breeding or in response to resource availability [33, 40, 54-55]. We therefore collected species-level foraging information from the EltonTraits database [56]. This database splits the overall resource use for each species into various percentages of fruit and nectar, vertebrate prey, and insects, and may therefore be a more ecologically 
realistic method of measuring the feeding niches of the species in this study. We also used this database to validate fine-scale niche assignments, such that bats assigned to a fine-scale category had to have at least $50 \%$ of their diet comprised of a single type of resource, and any bats whose diets were composed of approximately equal plant and animal material were assigned as "omnivores"(e.g., Phyllostomus discolor, whose diet is coded as 30\% invertebrates, $40 \%$ fruit, and 30\% nectar in EltonTraits).

\section{DNA extraction}

We performed all DNA isolations and library preparations in a UV-sterilized laminar flow hood to prevent aerosol contamination. We extracted total DNA from each guano sample using the QIAamp PowerFecal DNA Kit (MO BIO Laboratories, QIAGEN Co., Carlsbad, CA) following the manufacturer's instructions with the following alterations: prior to homogenization, we incubated fecal samples in the provided lysis solutions (Powerbead + Solution C1) for $10 \mathrm{~min}$ at $70{ }^{\circ} \mathrm{C}$. Next, we homogenized the fecal material in a Fisherbrand Bead Mill 24 homogenizer (Fisher Scientific, Pittsburgh, PA) at $6 \mathrm{~m} / \mathrm{s}$ for 1-2 min, until the fecal slurry was fully homogenized. At the elution step, we eluted with warmed PCR-grade water instead of the provided C6 buffer and incubated columns for two minutes prior to centrifugation. In addition to our samples, we extracted one "blank" (water only) sample to account for bacterial contamination of the extraction kit, which has been documented as an important source of error in other metagenomic studies $[57,58]$. As a positive control, we also extracted $25 \mu \mathrm{L}$ of genomic DNA from a mock microbial community of known composition (ZYMOBIOMICS, Zymo Research, Inc., Irvine, CA). Purified DNA extracts were preserved at $-25{ }^{\circ} \mathrm{C}$ prior to next generation sequencing (NGS) library preparation.

\section{Microbiota profiling}

For the Belize 2016-2017 samples, libraries targeting the V4 hypervariable region of the $16 \mathrm{~S}$ rRNA gene were amplified using primer pair 515F/806 [59, 60]. Amplicon libraries were generated and sequenced by MrDNA, using a single-end sequencing on an Illumina MiSeq platform (Shallowater, TX, USA). All 2018 fecal microbiome libraries were prepared and sequenced by the Integrated Microbiome Resource facility of Dalhousie University (Halifax, NS, Canada). Briefly, each 2018 fecal sample underwent PCR amplification of the V6-V8 hypervariable region of the $16 \mathrm{~S}$ rRNA gene using universal primers 969FB and 1406R [61]. Both 2016-2017 and 2018 libraries were paired-end sequenced $(2 \times 300 \mathrm{bp})$ on an Illumina MiSeq platform using V3 chemistry. While it is generally preferable to standardize primer target regions, our data were prepared for other studies by independent contributors and later collated for meta-analysis. Different primer regions have the ability to produce slightly different taxonomic assemblages, but in general, beta diversity metrics have been shown to be robust to both primer region and sequencing platform biases [62].

\section{Functional profiling}

We processed data generated from different sequencing runs separately using the QIIME2 v. 2019.10 pipeline of tools [63] We imported each dataset and performed quality filtering with the DADA2 plugin, which trims barcode and primer sequences, identifies and filter chimeric sequences, and calls amplicon sequence variants (ASVs) [64]. In general, we trimmed the first 10-20 base pairs to account for low-quality reads and truncated each dataset at the point where per-base quality score tapered to below roughly $\mathrm{Q}=20$. We then used the representative sequences as input for taxonomic classification using the naive Bayesian classifier trained on the SILVA 132 99\% OTUs database $[65,66]$. Each classifier was individually trained on the specific primer sets used in each study as recommended by the developers [67]. Because each dataset was prepared with a slightly different set of genetic protocols, we processed each one separately until taxonomic assignment was determined. After generating taxonomic feature tables for each dataset, we further filtered out mitochondrial and chloroplast reads from the datasets as well as any reads that could not be defined at least to the phylum level. Such sequences may represent novel bacteria not yet characterized in 16S rRNA gene databases and are of limited use for functional prediction, since functionally annotated whole genome databases are generally less complete than marker gene databases [68]. After filtering, all datasets were merged into a single feature table for functional profiling.

It is not possible to directly infer bacterial functions from marker gene inventories, so we used Phylogenetic Investigation of Communities by Reconstruction of Unobserved States (PICRUSt2) to predict metagenomic profiles for each microbiota sample $[69,70]$. PICRUSt2 works by first inserting observed $16 \mathrm{~S}$ rRNA gene sequences into a bacterial reference phylogeny, and then using hidden state prediction models to assign functions based on the closest matching bacterial reference genome [70]. The output of the algorithm reports an ASV abundance table normalized by predicted $16 \mathrm{~S}$ rRNA gene copy number for each ASV. We merged all ASV tables prior to PICRUSt2 inference to ensure that the same predictions would be output for the same ASVs present across multiple feature tables. 


\section{Statistical analyses}

Previous studies suggest that rarefying data to account for variable library depth is not appropriate [71], so instead of rarefying our data to an arbitrary subsampling depth, we performed a Hellinger transformation to scale the data using $\mathrm{R}$ package microbiome [72, 73]. We first tested if overall metagenome functional profiles differed according to host taxonomy and dietary ecology (both coarse and fine) using the adonis.pair function in $\mathrm{R}$ package Ecolltils and applying a Benjamini-Hochberg correction for multiple comparisons [74]. Using R package phyloseq [75], we performed PERMANOVA tests on Bray-Curtis distances of metagenomes as a function of host identity and diet, taking into account the nested nature of host taxonomy [study.bray $\sim$ FeedingNiche ${ }^{*}$ HostSpecies + HostGenus + HostFamily]. Next, we performed paired PERMANOVAs to test for differences between each individual feeding niche.

PERMANOVA can detect differences between groups of data, but the test operates on distance matrices and therefore cannot determine which specific functions are driving group differences. To test for differential enrichment of specific metagenome functions, we performed Linear Discriminant Analysis Effect Size (LEfSe) analysis as implemented on the Galaxy platform (https://hutte nhower.sph.harvard.edu/galaxy/) [76]. We grouped samples by feeding niche in both coarse (animalivores, herbivores) and fine (frugivores, insectivores, omnivores, carnivores, and sanguivores) ecological classification schemes, and set the LDA score cutoff to 2.5 to impose a strict effect size criterion on differentially abundant features. Due to the low number of omnivore observations, we grouped them together with the animalivorous bats for the coarse LEfSe analysis based on prior knowledge that these species rely heavily on insects during some seasons [39, 42, 77].

We also sought to assess the influence of diet and host phylogeny on predicted microbiome functions by representing these values as continuous traits. We merged metagenome functions by host species and computed the Bray-Curtis distances for all species. For the host phylogeny, we computed patristic distances (i.e., the sum of the branch lengths linking two nodes) between terminal taxa using a pruned phylogeny from Upham et al. 2019 [78]. We reconciled taxonomic changes between the sampled species and their closest synonymous or sister taxon represented in the Upham dataset using batnames.org (Additional file 2: Table S1) [79]. For each of these same taxa, we also collected species-level dietary data from the EltonTraits database [56], which represents mammalian diets as percentages of various food resources (vertebrates, insects, nectar, fruit, etc.). We transformed these proportional data into a distance matrix using the function "dist.prop" in R package ade4 using the "Manly" method [80, 81]. Using these matrices, we tested for associations between gut microbiome functions and host phylogeny and diet using multiple regression on matrices (MRM) implemented in the $\mathrm{R}$ package ecodist using the formula merged.functional.dist $\sim$ bat.diets.dist + PatristicDistMatrix [82]. Because bat microbiomes are known to be highly variable among individuals of the same species $[11,43]$, we also tested the predictive power of host diet using random forests on the full per-individual dataset. We first removed any features from the dataset that were present in fewer than $10 \%$ of samples and scaled all raw counts by transforming to Z-scores. Finally, we constructed random forest classifiers using $\mathrm{R}$ package randomForest to test the ability of the functional profiles of each sample to predict the coarse or fine niche of the host [83, 84]. Each classifier was built over 10,000 trees and out-of-bag error rate (OOB\%) was estimated for each model. Model significance and accuracy was further evaluated using permutation testing and cross-validation, respectively.

We tested for evolutionary signal in microbiome functions by treating each discriminatory functional pathway identified by the random forest analysis as a trait of the host following an approach similar to that used by Capunitan et al. (2020) [47]. We used a pruned species-level phylogeny of bats from Upham et al. 2019 [78]. Because host taxonomy changes frequently, we manually correct species names to reflect the most up-to-date taxonomy and to reconcile ambiguous host species identities (Additional file 2: Table S1). Microbiome traits were averaged across individuals of the same species using "merge_samples" prior to undergoing center-log transform and matched to the tips in the phylogeny using the "treedata" function. Using the "fitContinuous" function in geiger [85], we tested the fit of Brownian Motion, Ornstein-Uhlenbeck (OU; single optimum), Early Burst, and White Noise models and compared them using weighted Akaike information criterion (AIC). Akaike weights were calculated from AIC scores using the "aicw" function. As a measure of phylogenetic signal, we calculated Pagel's lambda $(\lambda)$ [86], which is a scaling parameter that ranges from 0 (no phylogenetic signal) to 1 (strong phylogenetic signal).

\section{Abbreviations}

AMNH: American Museum of Natural History; ASV: Amplicon sequence variant; BM: Brownian Motion; EB: Early Burst; LefSE: Linear discriminant analysis Effect Size; MRM: Multiple regression on matrices; NGS: Next-generation sequencing; OOB: Out-of-bag error; OU: Ornstein-Uhlenbeck; PERMANOVA: Permutational multivariate analysis of variance. 


\section{Supplementary Information}

The online version contains supplementary material available at https://doi. org/10.1186/s42523-021-00139-8.

Additional file 1: Fig. S1. Principal coordinates analysis of predicted bat microbiome functions colored according to coarse host niche.

Additional file 2: Table S1. Table of species sampled in this study and their closest relative in the Upham et al. 2019 phylogeny. Patristic distances were computed using the closest terminal taxon available in this phylogeny, and taxon names for the MRMs were coerced to match those in the phylogeny. Two species in the Lutz et al. dataset were not identified beyond genus; for these, we chose a congeneric species known to occur in the sampled localities for use in patristic distance calculations.

Additional file 3: Table S2. Sample metadata and study provenance for all bat microbiomes used in this study.

Additional file 4: Table S3. Confusion matrix for Random Forest models attempting to predict host family membership based on metagenome functions. Out-of-bag error rate was 49.36\%.

Additional file 5: Table S4. Confusion matrix for Random Forest models attempting to predict host genus membership based on metagenome functions. Out-of-bag error rate was 58.35\%.

\section{Acknowledgements}

We would like to thank M. Brock Fenton, M. Howells, N. Duncan, and the Lamanai Bat-a-thon team for help catching animals during the fieldwork portion of this study, as well as the Belize Forestry Department for granting us permission to conduct this research. We acknowledge the Yucatec Maya people as the traditional residents of Orange Walk District, Belize and thank them for their continued stewardship of the forests surrounding our study sites. We thank K. Kohl and S. Galen for helpful conversations on handling data, as well as $\mathrm{H}$. Lutz and J. Gilbert for feedback on the preprint which improved the quality of the manuscript. We also thank two anonymous reviewers whose suggestions greatly improved the writing and clarity of the study.

\section{Authors' contributions}

MRI, NBS, and SLP conceived of and designed the study. MRI, MD, and NBS performed the fieldwork and collected samples. MD and MRI completed the laboratory work, and CW and KK advised MRI on methods and bioinformatics. MRI wrote the manuscript. All authors read and approved the final manuscript.

\section{Funding}

This work was supported by the following grants awarded to MRI: an Explorer's Club Exploration Grant, a Grant-in-Aid of Research from the American Society of Mammalogists, a Grant-in-aid from Sigma Xi, and a Grant-in-Aid from the Society for Integrative and Comparative Biology. Fieldwork was funded by the Taxonomic Mammalogy Fund of the Department of Mammalogy, the Richard Gilder Graduate School at the American Museum of Natural History, and a CSU-AAUP travel grant awarded to MD. MRI was also supported by a Graduate Research Assistantship awarded by the Richard Gilder Graduate School.

\section{Availability of data and materials}

All Belize 2016, 2017, and 2018 raw 16S rRNA gene sequences are publicly available at the NCBI SRA under BioProject PRJNA701438. Statistical packages and full code are available at https://github.com/Melissalngala/BatFunx. Additional metadata files can be accessed on FigShare:(https://figshare.com/proje cts/You_Are_More_Than_What_You_Eat_Differential_Enrichment_of_Micro biome_Functions_Across_Bat_Dietary_Niches/98408). Data from previous studies has been made publicly available by the authors.

\section{Declarations}

Ethics approval and consent to participate Not applicable.

\section{Consent for publication}

Not applicable.

\section{Competing interests}

The authors declare that they have no competing interests.

\section{Author details}

${ }^{1}$ Department of Vertebrate Zoology, National Museum of Natural History, Washington, DC, USA. ${ }^{2}$ Department of Mammalogy, The American Museum of Natural History, New York, NY, USA. ${ }^{3}$ Division of Invertebrate Zoology, The American Museum of Natural History, New York, NY, USA. ${ }^{4}$ Department of Biological Sciences, Southern Connecticut State University, New Haven, CT, USA. ${ }^{5}$ Sackler Institute for Comparative Genomics, The American Museum of Natural History, New York, NY, USA. ${ }^{6}$ Bioinformatics and Computational Genomics Laboratory, Hunter College, City University of New York, New York, NY, USA. ${ }^{7}$ Department of Biological Sciences, Hunter College, City University of New York, New York, NY, USA. ${ }^{8}$ Institute of Computational Biomedicine, Weill Cornell Medical College, New York, NY, USA

Received: 1 April 2021 Accepted: 20 October 2021

Published online: 14 December 2021

\section{References}

1. Kohl KD, Oakeson KF, Orr TJ, Miller AW, Forbey JS, Phillips CD, et al. Metagenomic sequencing provides insights into microbial detoxification in the guts of small mammalian herbivores (Neotoma spp). FEMS Microbiol Ecol. 2018;42:319.

2. Lisandra Zepeda Mendoza M, Xiong Z, Escalera-Zamudio M, Kathrine Runge A, Thézé J, Streicker D, et al. Hologenomic adaptations underlying the evolution of sanguivory in the common vampire bat. Nat Ecol Evol. 2018;2:659-68

3. Godoy-Vitorino F, Goldfarb KC, Karaoz U, Leal S, Garcia-Amado MA, Hugenholtz $\mathrm{P}$, et al. Comparative analyses of foregut and hindgut bacterial communities in hoatzins and cows. ISME J. 2012:6:531-41.

4. Groussin M, Mazel F, Sanders JG, Smillie CS, Lavergne S, Thuiller W, et al. Unraveling the processes shaping mammalian gut microbiomes over evolutionary time. Nat Commun. 2017. https://doi.org/10.1038/ncomm s14319.

5. Carrillo-Araujo M, Tas N, Alcantara-Hernandez RJ, Gaona O, Schondube JE, Medellin RA, et al. Phyllostomid bat microbiome composition is associated to host phylogeny and feeding strategies. Front Microbiol. 2015. https://doi.org/10.3389/fmicb.2015.00447.

6. Amato KR, Sanders JG, Song SJ, Nute M, Metcalf JL, Thompson LR, et al. Evolutionary trends in host physiology outweigh dietary niche in structuring primate gut microbiomes. ISME J. 2019;13:576-87.

7. McKenney EA, Rodrigo A, Yoder AD. Patterns of gut bacterial colonization in three primate species. PLoS ONE. 2015. https://doi.org/10.1371/journal. pone.0124618.

8. Numberger D, Herlemann DPR, Jürgens K, Dehnhardt G, Schulz-Vogt H. Comparative analysis of the fecal bacterial community of five harbor seals (Phoca vitulina). MicrobiologyOpen. 2016;5:782-92.

9. Martiny JBH, Lennon JT, Martiny AC, Martiny JBH, Jones SE, Lennon JT, et al. Microbiomes in light of traits: a phylogenetic perspective. Science. 2015;350:6261.

10. Louca S, Parfrey LW, Doebeli M. Decoupling function and taxonomy in the global ocean microbiome. Science. 2016;353:1272-7.

11. Lutz HL, Jackson EW, Webala PW, Babyesiza WS, Kerbis Peterhans JC, Demos TC, et al. Ecology and host identity outweigh evolutionary history in shaping the bat microbiome. mSystems. 2019;4:e00511-19.

12. Song SJ, Sanders JG, Delsuc F, Metcalf J, Amato K, Taylor MW, et al. Comparative analyses of vertebrate gut microbiomes reveal convergence between birds and bats. MBio. 2020;11:e02901-19.

13. Phillips CD, Hanson J, Wilkinson JE, Koenig L, Rees E, Webala P, et al. Microbiome structural and functional interactions across host dietary Niche space. Integr Comp Biol. 2017;57:743-55.

14. Amato KR, Yeoman CJ, Kent A, Righini N, Carbonero F, Estrada A, et al. Habitat degradation impacts black howler monkey (Alouatta pigra) gastrointestinal microbiomes. ISME J. 2013;716:1344-53.

15. Ingala MR, Simmons NB, Perkins $L$. Bats are an untapped system for understanding microbiome evolution in mammals. mSphere. 2018;3:1-6. 
16. Clayton JB, Vangay P, Huang H, Ward T, Hillmann BM, Al-Ghalith GA, et al. Captivity humanizes the primate microbiome. Proc Natl Acad Sci. 2016;113:201521835

17. Mccord Al, Chapman CA, Weny G, Tumukunde A, Hyeroba D, Klotz K, et al. Fecal microbiomes of non-human primates in Western Uganda reveal species-specific communities largely resistant to habitat perturbation. Am J Primatol. 2014. https://doi.org/10.1002/ajp.22238.

18. Gomez A, Petrzelkova K, Yeoman CJ, Vlckova K, Mrázek J, Koppova I, et al. Gut microbiome composition and metabolomic profiles of wild western lowland gorillas (Gorilla gorilla gorilla) reflect host ecology. Mol Ecol. 2015. https://doi.org/10.1111/mec.13181.

19. Kohl KD, Denise DM. The woodrat gut microbiota as an experimental system for understanding microbial metabolism of dietary toxins. Front Microbiol. 2016. https://doi.org/10.3389/fmicb.2016.01165.

20. Baxter NT, Wan JJ, Schubert AM, Jenior ML, Myers P, Schloss PD. Intra- and interindividual variations mask interspecies variation in the microbiota of sympatric Peromyscus populations. Appl Environ Microbiol. 2015;81:396-404.

21. Simmons NB, Conway T. Evolution of ecological diversity in bats. In: Kunz TH, Fenton MB, editors. Bat ecology. University of Chicago Press; 2003. p. 493-535.

22. Teeling EC, Springer MS, Madsen O, Bates P, O'Brien SJ, Murphy WJ. A molecular phylogeny for bats illuminates biogeography and the fossil record a molecular phylogeny for bats illuminates biogeography and the fossil record. Science. 2009;307:580-4.

23. Shi JJ, Rabosky DL. Speciation dynamics during the global radiation of extant bats. Evolution. 2015;6:1528-45.

24. Akaike H. Maximum likelihood identification of Gaussian autoregressive moving average models. Biometrika. 1973;60:255-65.

25. Uhlenbeck GE, Ornstein LS. On the theory of the Brownian motion. Phys Rev. 1930;36:823.

26. Metges CC. Contribution of microbial amino acids to amino acid homeostasis of the host. J Nutr. 2000. https://doi.org/10.1093/jn/130.7.1857S

27. Neis EPJG, Dejong CHC, Rensen SS. The role of microbial amino acid metabolism in host metabolism. Nutrients. 2015. https://doi.org/10.3390/ nu7042930.

28. Wendeln MC, Runkle JR, Kalko E. Nutritional values of 14 Fig species and bat feeding preferences in Panama. Biotropica. 2000;32:489-501.

29. Thomas DW. Fruit intake and energy budgets of frugivorous bats. Physiol Zool. 1984. https://doi.org/10.1086/physzool.57.4.30163347.

30. Newsome SD, Feeser KL, Bradley CJ, Wolf C, Takacs-Vesbach C, Fogel ML. Isotopic and genetic methods reveal the role of the gut microbiome in mammalian host essential amino acid metabolism. Proc R Soc B Biol Sci. 2020. https://doi.org/10.1098/rspb.2019.2995.

31. Estes AM, Hearn DJ, Agrawal S, Pierson EA, Hotopp JCD. Comparative genomics of the Erwinia and Enterobacter olive fly endosymbionts. Sci Rep. 2018:8:1-13.

32. Hobson KA, Leticia MM, Nicte RP, Germán MC, VÍctor S-C, Gerardo Herrera $L M$, et al. Sources of protein in two species of phytophagous bats in a seasonal dry forest: evidence from stable-isotope analysis. J Mammal. 2001;82:352-61.

33. Dinerstein E. Reproductive ecology of fruit bats and the seasonality of fruit production in a Costa Rican Cloud Forest. Biotropica. 1986;18:307-18.

34. Smith EA, Macfarlane GT. Enumeration of amino acid fermenting bacteria in the human large intestine: effects of $\mathrm{pH}$ and starch on peptide metabolism and dissimilation of amino acids. FEMS Microbiol Ecol. 1998:25:355-68.

35. Ingala MR, Becker DJ, Holm JB, Kristiansen K, Simmons NB. Habitat fragmentation is associated with dietary shifts and microbiota variability in common vampire bats. Ecol Evol. 2018;2019:1-16.

36. Breidenstein CP. Digestion and assimilation of bovine blood by a vampire bat (Desmodus rotundus). J Mammal. 2010;63:482-4.

37. Voigt CC, Sörgel K, Dechmann DKN. Refueling while flying: foraging bats combust food rapidly and directly to power flight. Ecology. 2010. https:// doi.org/10.1890/09-2232.1.

38. Voigt CC, Sörgel K, Šuba J, Keišs O, Petersons G. The insectivorous bat Pipistrellus nathusii uses a mixed-fuel strategy to power autumn migration. Proc R Soc B Biol Sci. 2012. https://doi.org/10.1098/rspb.2012.0902.

39. Clare EL, Goerlitz HR, Drapeau VA, Holderied MW, Adams AM, Nagel J, et al. Trophic niche flexibility in Glossophaga soricina: how a nectar seeker sneaks an insect snack. Funct Ecol. 2014;28:632-41.
40. Oelbaum PJ, Fenton MB, Simmons NB, Broders HG. Community structure of a Neotropical bat fauna as revealed by stable isotope analysis: not all species fit neatly into predicted guilds. Biotropica. 2019. https://doi.org/ 10.1111/btp.12700.

41. Bearhop S, Adams CE, Waldron S, Fuller RA, Macleod H. Determining trophic niche width: a novel approach using stable isotope analysis. J Anim Ecol. 2004. https://doi.org/10.1111/j.0021-8790.2004.00861.x.

42. Willig MR, Camilo GR, Noble SJ. Dietary overlap in frugivorous and insectivorous bats from Edaphic Cerrado habitats of Brazil. J Mammal. 1993. https://doi.org/10.2307/1381910.

43. Ingala MR, Simmons NB, Wultsch C, Krampis K. Comparing microbiome sampling methods in a wild mammal : fecal and intestinal samples record different signals of host ecology, evolution. Front Microbiol. 2018. https:// doi.org/10.3389/fmicb.2018.00803.

44. Martins EP. Estimating the rate of phenotypic evolution from comparative data. Am Nat. 1994. https://doi.org/10.1086/285670.

45. Grieneisen L, Dasari M, Gould TJ, Björk JR, Grenier J-C, Yotova V, et al. Gut microbiome heritability is nearly universal but environmentally contingent. Science. 2021;373:181-6.

46. Adair KL, Douglas AE. Making a microbiome: the many determinants of host-associated microbial community composition. Curr Opin Microbiol. 2017;35:23-9.

47. Capunitan DC, Johnson O, Terrill RS, Hird SM. Evolutionary signal in the gut microbiomes of 74 bird species from Equatorial Guinea. Mol Ecol. 2020. https://doi.org/10.1111/mec.15354.

48. Legrand T, Wos-Oxley ML, Wynne JW, Weyrich LS, Oxley APA. Dead or alive: microbial viability treatment reveals both active and inactive bacterial constituents in the fish gut microbiota. J Appl Microbiol. 2021. https:// doi.org/10.1111/jam.15113.

49. Chen MX, Wang SY, Kuo CH, Tsai IL. Metabolome analysis for investigating host-gut microbiota interactions. J Formosan Med Assoc. 2019. https:// doi.org/10.1016/j.jfma.2018.09.007.

50. Wilkinson GS, South JM. Life history, ecology and longevity in bats. Aging Cell. 2002;1:124-31

51. Phillips CD, Phelan G, Dowd SE, McDonough MM, Ferguson AW, Delton Hanson J, et al. Microbiome analysis among bats describes influences of host phylogeny, life history, physiology and geography. Mol Ecol. 2012:21:2617-27.

52. Gilbert JA, Jansson JK, Knight R. The Earth Microbiome project: successes and aspirations. BMC Biol. 2014;12:69-69.

53. Sikes RS, Bryan JA II, Byman D, Danielson BJ, Eggleston J, Gannon MR, et al. 2016 Guidelines of the American Society of Mammalogists for the use of wild mammals in research and education. J Mammal. 2016;97:663-88.

54. Ingala MR, Simmons NB, Wultsch C, Krampis K, Provost KL, Perkins SL. Molecular diet analysis of neotropical bats based on fecal DNA metabarcoding. Ecol Evol. 2021. https://doi.org/10.1002/ece3.7579.

55. Darosci AAB, Bruna EM, Motta-Junior JC, da Silva Ferreira C, Blake JG, Munhoz CBR. Seasonality, diaspore traits and the structure of plant-frugivore networks in Neotropical savanna forest. Acta Oecol. 2017;84:15-22.

56. Wilman H, Belmaker J, Simpson J, de la Rosa C, Rivadeneira MM, Jetz W. EltonTraits 1.0: species-level foraging attributes of the world's birds and mammals. Ecology. 2014. https://doi.org/10.1890/13-1917.1.

57. Mohammadi T, Reesink HW, Vandenbroucke-Grauls CM, Savelkoul PH. Removal of contaminating DNA from commercial nucleic acid extraction kits. J Microbiol Methods. 2005;61:285-8.

58. Glassing A, Dowd SE, Galandiuk S, Davis B, Chiodini RJ. Inherent bacterial DNA contamination of extraction and sequencing reagents may affect interpretation of microbiota in low bacterial biomass samples. Gut Pathogens. 2016;8:24.

59. Caporaso JG, Lauber CL, Walters WA, Berg-Lyons D, Lozupone CA, Turnbaugh PJ, et al. Global patterns of 165 rRNA diversity at a depth of millions of sequences per sample. Proc Natl Acad Sci USA. 2011;108(Supplement_1):4516-22.

60. Apprill A, Mcnally S, Parsons R, Weber L. Minor revision to V4 region SSU rRNA 806R gene primer greatly increases detection of SAR11 bacterioplankton. Aquat Microb Ecol. 2015;75:129-37.

61. Comeau AM, Li WKW, Tremblay JÉ, Carmack EC, Lovejoy C. Arctic ocean microbial community structure before and after the 2007 record sea ice minimum. PLOS ONE. 2007;2011:6 
62. Tremblay J, Singh K, Fern A, Kirton ES, He S, Woyke T, et al. Primer and platform effects on 16S rRNA tag sequencing. Front Microbiol. 2015. https://doi.org/10.3389/fmicb.2015.00771.

63. Rideout JR, Dillon MR, Bokulich NA, Abnet CC, Gabriel A, Ghalith A, et al. QIIME 2: Reproducible, interactive, scalable, and extensible microbiome data science. PeerJ Preprints. 2018.

64. Callahan BJ, McMurdie PJ, Rosen MJ, Han AW, Jo A Johnson A, Holmes SP. DADA2: high resolution sample inference from Illumina amplicon data. Nat Methods. 2016:13:48-56.

65. Bokulich NA, Kaehler BD, Rideout JR, Dillon M, Bolyen E, Knight R, et al. Optimizing taxonomic classification of marker-gene amplicon sequences with QIIME 2's q2-feature-classifier plugin. Microbiome. 2018;6:1-17.

66. Quast C, Pruesse E, Yilmaz P, Gerken J, Schweer T, Yarza P, et al. The SILVA ribosomal RNA gene database project: improved data processing and web-based tools. Nucleic Acids Res. 2013. https://doi.org/10.1093/nar/ gks1219.

67. Wang Q, Garrity GM, Tiedje JM, Cole JR. Naïve Bayesian classifier for rapid assignment of rRNA sequences into the new bacterial taxonomy. Appl Environ Microbiol. 2007;73:5261-7.

68. Tessler M, Neumann JS, Afshinnekoo E, Pin M, Hersch R, Velho LFM, et al. Large-scale differences in microbial biodiversity discovery between 165 amplicon and shotgun sequencing. Sci Rep. 2017;7:1-14.

69. Langille MGI, Zaneveld J, Caporaso JG, McDonald D, Knights D, Reyes JA et al. Predictive functional profiling of microbial communities using $16 \mathrm{~S}$ rRNA marker gene sequences. Nat Biotechnol. 2013;31:814-21.

70. Douglas GM, Maffei VJ, Zaneveld J, Yurgel SN, Brown JR, Taylor CM, et al. PICRUSt2: an improved and extensible approach for metagenome inference. bioRxiv. 2019;8:497.

71. McMurdie PJ, Holmes S. Waste not, want not: why rarefying microbiome data is inadmissible. PLoS Comput Biol. 2014;10:e1003531.

72. Legendre P, Gallagher ED. Ecologically meaningful transformations for ordination of species data. Oecologia. 2001. https://doi.org/10.1007/ s004420100716.

73. Lahti L, Shetty S, Blake T. Tools for microbiome analysis in R. Microbiome Package Version 099. 2017.

74. Salazar G. EcolUtils: utilities for community ecology analysis. 2019.

75. McMurdie PJ, Holmes S. Phyloseq: an R package for reproducible interactive analysis and graphics of microbiome census data. PLoS ONE. 2013;8:e61217.

76. Segata N, Izard J, Waldron L, Gevers D, Miropolsky L, Garrett WS, et al. Metagenomic biomarker discovery and explanation. Genome Biol. 2011. https://doi.org/10.1186/gb-2011-12-6-r60.

77. Herrera LG, Gutierrez E, Hobson KA, Altube B, Díaz WG, Sánchez-Cordero V. Sources of assimilated protein in five species of new world frugivorous bats. Oecologia. 2002;133:280-7.

78. Upham NS, Esselstyn JA, Jetz W. Inferring the mammal tree: species-level sets of phylogenies for questions in ecology, evolution, and conservation. PLoS Biol. 2019. https://doi.org/10.1371/journal.pbio.3000494.

79. Simmons NB, Cirranello AL. Bat species of the world: a taxonomic and geographic database. https://batnames.org/. Accessed 21 Sept 2021.

80. Dray S, Dufour AB. The ade4 package: implementing the duality diagram for ecologists. J Stat Softw. 2007. https://doi.org/10.18637/jss.v022.104.

81. Cooper Rl, Manly BFJ. Multivariate statistical methods: a primer. J R Stat Soc Ser A (Gen). 1987. https://doi.org/10.2307/2982053.

82. Goslee S, Urban D. Package' ecodist '.R topics documented. 2015.

83. Liaw A, Yan J, Li W, Han L, Schroff F, Criminisi A, et al. Package'randomForest.'R news. 2014

84. Breiman L. Random forests. In: Machine learning. 2001.

85. Pennell MW, Eastman JM, Slater GJ, Brown JW, Uyeda JC, Fitzjohn RG, et al. Geiger v2.0: an expanded suite of methods for fitting macroevolutionary models to phylogenetic trees. Bioinformatics. 2014. https://doi.org/10. 1093/bioinformatics/btu181.

86. Pagel M. Inferring the historical patterns of biological evolution. Nature. 1999. https://doi.org/10.1038/44766.

\section{Publisher's Note}

Springer Nature remains neutral with regard to jurisdictional claims in published maps and institutional affiliations.

Ready to submit your research? Choose BMC and benefit from:

- fast, convenient online submission

- thorough peer review by experienced researchers in your field

- rapid publication on acceptance

- support for research data, including large and complex data types

- gold Open Access which fosters wider collaboration and increased citations

- maximum visibility for your research: over $100 \mathrm{M}$ website views per year

At BMC, research is always in progress.

Learn more biomedcentral.com/submissions 